\title{
One-Pot Catalytic Enantioselective Synthesis of 2-Pyrazolines
}

\author{
Connor J. Thomson ${ }^{[a]}$, David M. Barber ${ }^{[b]}$ and Darren J. Dixon ${ }^{[*][a]}$
}

\begin{abstract}
A scalable, one-pot, enantioselective catalytic synthesis of 2-pyrazolines from beta-substituted enones and hydrazines is described. Pivoting on a two stage catalytic Michael addition / condensation strategy, the use of an aldehyde to generate a suitable hydrazone derivative of the hydrazine was found to be key for curtailing background reactivity and tuning the catalyst-controlled enantioselectivity. The new synthetic protocol is easy to perform, uses a new and readily prepared cinchona-derived bifunctional catalyst, is broad in scope, and tolerates a range of functionalities with high enantioselectivity (up to $>99: 1$ er). The significant scalability of this methodology has been demonstrated with the synthesis of $>80$ grams of a pyrazoline product with $89 \%$ catalyst recovery.
\end{abstract}

Heterocycles containing an N-N bond are abundant in natural products, synthetic therapeutics, and agrochemicals. ${ }^{[1]}$ In particular, optically active pyrazolines and their derivatives are in high demand for their intrinsic biological properties. ${ }^{[2]}$ 2Pyrazolines specifically find widespread use in small-molecule drugs, agrochemicals, and have been noted for their valuable properties in material science (Scheme 1). ${ }^{[3]}$ Furthermore, the use of 2-pyrazolines as valuable intermediates in the synthesis of cyclopropanes and pyrazoles is well-documented. ${ }^{[4]}$

Although there are many examples describing the synthesis of 2-pyrazolines as racemates, methods for the efficient preparation of enantiomerically enriched 2-pyrazolines remain limited. ${ }^{[5]}$ In 2000, Kanai reported the first enantioselective synthesis of 2-pyrazolines, using a Lewis acid catalyzed 1,3-dipolar cycloaddition. ${ }^{[6]}$ This was subsequently followed by the acid catalyzed electrocyclization methodology of List, and independent reports from Cordova and Carillo on the synthesis of 2-pyrazolines via pyrazolidines. ${ }^{[7]}$ Brière also disclosed the elegant phase-transfer catalyzed addition of carbazates to chalcones. ${ }^{[8]}$ Deng later reported the use of benzyl-protected carbazates in the enantioselective synthesis of 2-pyrazolines (Scheme 1B)..$^{\text {[9] }}$

Despite these elegant examples, we were attracted to the challenge of developing a broadly applicable platform for the metal-free, expeditious synthesis of enantioenriched 2pyrazolines. Whilst the use of hydrazine nucleophiles in the synthesis of racemic pyrazolines is well-established, the high reactivity of these reagents has, to date, restricted the development of a corresponding enantioselective method. ${ }^{[10]}$ As a strategy to circumvent their high inherent nucleophilicity, we envisaged the use of an aldehyde to generate a suitable hydrazone derivative to impart tunability and dampen reactivity of the hydrazine moiety (Scheme 1B). ${ }^{[11]}$ Such an approach could grant stereoselective access to enantioenriched 2pyrazolines after in-situ cleavage of the hydrazone and subsequent intramolecular condensation (Scheme 1C). Herein we wish to report our findings.

The reaction of methylhydrazine-derived hydrazone $\mathbf{1 a}$

[a] C. J. Thomson, Prof. Dr. D. J. Dixon, Department of Chemistry, Chemistry Research Laboratory, University of Oxford, United Kingdom, Mansfield Road, Oxford OX1 3TA

[b] Dr. D. M. Barber, Research \& Development, Weed Control Chemistry Bayer AG, Crop Science Division, Industriepark Höchst, Frankfurt am Main, 65926, Germany

[*] darren.dixon@chem.ox.ac.uk
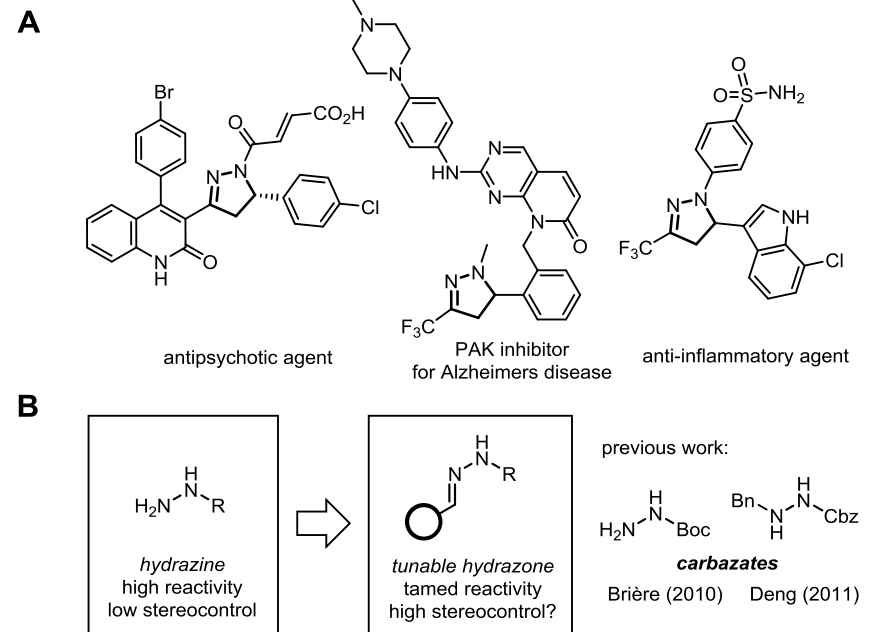

C

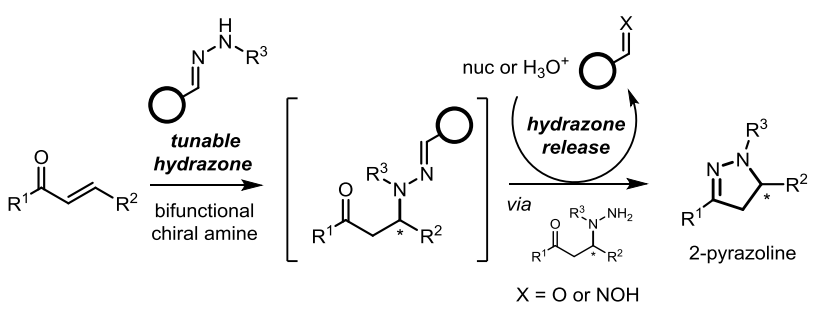

Scheme 1. (A) Bioactive pyrazolines and their uses; (B) Rationale for use of protected hydrazines and previous work with carbazates; (C) Platform for azaMichael addition of the hydrazine sython enabled by a cleavable hydrazone.

with chalcone $\mathbf{2 a}$ was chosen as a model system and we hypothesized that bifunctional Brønsted base/H-bond donor catalysts could affect the desired enantioselective aza-Michael addition. ${ }^{[12]}$ A screen of potential catalysts revealed the cinchona family to be effective, with quinidine (Figure 1, catalyst A) offering promising selectivity; aza-Michael adduct $\mathbf{3 a}$ was obtained in 71:29 er. Encouraged by the early success with quinidine as catalyst, other cinchona-derived systems were investigated (Table 1A, entries 1-6). Use of the O-benzyl catalyst B resulted in a selectivity decrease, while bifunctional urea $\mathbf{C}$ showed an enhancement to 80:20 er. Interestingly, the formation of Michael adduct 4 was observed as a competitive side-product in some cases. ${ }^{[13]}$ Despite this, selectivity for $3 \mathbf{3}$ was improved with the use of catalyst D. Enantioselectivity was further improved with amide $\mathbf{E}$, suggesting the need for a single $\mathrm{H}$-bond donor for appropriate organization of the hydrazone-chalcone transition state. A screen of various amide groups revealed 3,5dichlorobenzoylamide catalyst $\mathbf{F}$ to be optimal (see ESI).

Our attention then turned to the structure of the hydrazone and its influence on the stereochemical outcome of the reaction. The presence of a methyl group at the 2,3 , or 4 positions of the arene ring of 1 all improved enantioselectivity (see Table $1 \mathrm{~A}$ and ESI for details). Pleasingly, 4-tert-butyl benzaldehyde was found to give the most substantial enantioselectivity when compared with benzaldehyde (entry 9, 90:10 er). Lowering the reaction temperature to $-15{ }^{\circ} \mathrm{C}$ and extending the reaction time to 48 hours further improved the er and conversion, to 95:5 er and $93 \%$, respectively. Furthermore, quantitative in situ cleavage of the chiral hydrazone intermediate (thereby releasing oxime $\mathbf{6 b}$ ) 


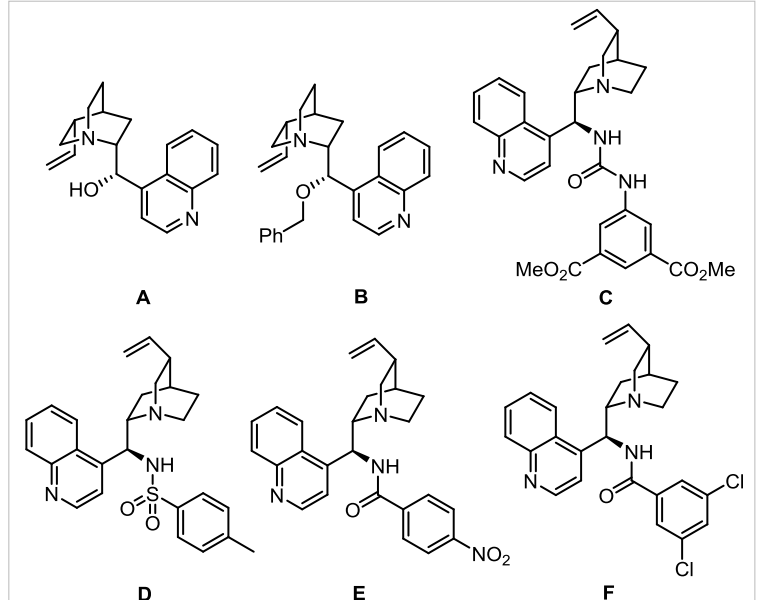

Figure 1. Selected catalysts investigated for the aza-Michael addition of 1a-d to chalcone 2.

could be achieved using hydroxylamine, without compromising the enantioselectivity (see Entry 4, Table 1B).

Combining these optimized transformations delivered a streamlined one-pot route to 2-pyrazolines from methylhydrazine, which was then ready for assessment of its scope. When substituents on both aromatic rings of the chalcone were varied, minimal fluctuation in enantioselectivity was observed across a range of electron-rich and electron-poor substrates (Scheme 2A). Potentially incompatible functionalities such as cyano, vinyl and ester groups were all well-tolerated. Difluorocyclopropane $\mathbf{5 l}$ and benzodioxole $\mathbf{5 q}$ are particularly appealing, with potential applications in medicinal chemistry programs. ${ }^{[14]}$ Substrates derived from alkyl aldehydes were compatible with this chemistry, giving excellent er's of up to $>99: 1$ for hexyl pyrazoline $5 w$ ( $82 \%$ yield). Ynenone $\mathbf{5 z}$, enynone $5 \mathbf{x}$ and alkenyl enone $\mathbf{5 y}$ were successfully employed to afford the corresponding alkynyl and alkenyl scaffolds in moderate to excellent er. Notably, heterocycles were competent substrates; furnishing the pyrazine, pyridine and thiophene structures $\mathbf{5 r - 5 t}$. Furthermore, variation of the hydrazine moiety afforded the $\mathrm{N}$ ethyl (5ab), $N$-cyanoethyl (5ac) and N-benzyl (5ad) 2pyrazolines in good yield and er at reaction rates similar to the methyl hydrazine system, thus demonstrating wider applicability of this chemistry. ${ }^{[15]}$

In order to explore the scalability of this methodology, we performed a model reaction with enone $\mathbf{2 b}$ on 100 gram scale. To ensure affordability and practicality upon scale-up, the reaction was performed at room temperature, rather than $-15{ }^{\circ} \mathrm{C}$, and benzaldehyde was employed as the hydrazone precursor instead of tert-butyl benzaldehyde. Smooth reactivity and a clean reaction profile allowed the isolation of 84.9 grams of pyrazoline $\mathbf{5 b}$ in $77 \%$ yield and $>99.9: 0.1$ er after a single recrystallization. The mild protocol allowed $\mathbf{5 b}$ to be cleanly isolated without the need for silica gel chromatography. Importantly, $89 \%$ of catalyst $\mathbf{F}$ was recovered in addition to $97 \%$ isolation of the benzaldehyde oxime by-product. ${ }^{[16]}$ The recovered catalyst was reused (on $0.5 \mathrm{mmol}$ scale) with no loss of reactivity or selectivity (see ESI).

With the aim of demonstrating the broader utility of our platform, pyrazoline $\mathbf{5 b}$ was explored as a scaffold for late stage

\begin{tabular}{cccccc}
\hline & & & & & \\
\hline
\end{tabular}

B Optimization of hydrazone cleavage release

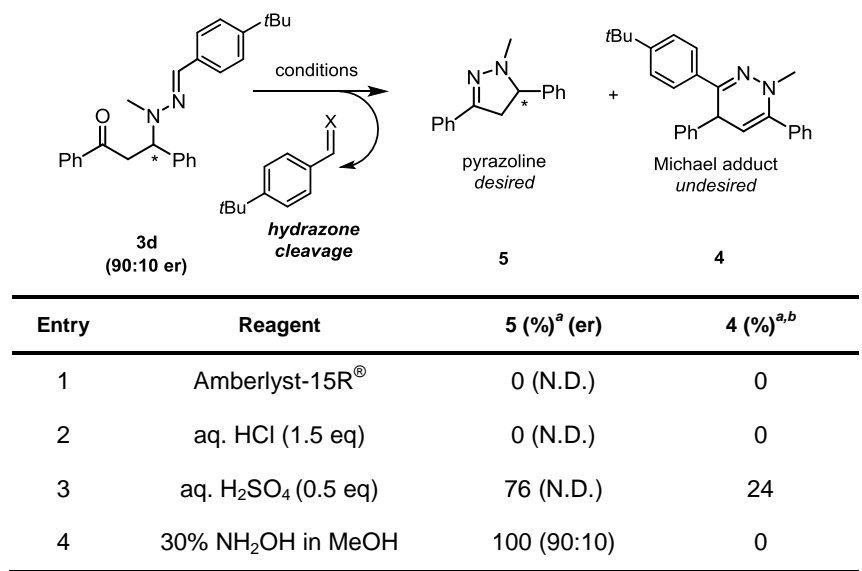

Table 1. (A) Optimization of the organocatalyzed aza-Michael addition; (B) Optimization of conditions for hydrazone cleavage. ${ }^{a}$ determined by ${ }^{1} \mathrm{H}$ NMR. ${ }^{b} \mathbf{4}$ was always obtained as a racemic mixture. ${ }^{c} 10 \mathrm{~mol} \%$ of catalyst $\mathbf{F}$ was used. ${ }^{d}$ performed at $-15{ }^{\circ} \mathrm{C}$ for 48 hours with $10 \mathrm{~mol} \%$ catalyst F. ${ }^{e}$ performed for 7 days. N.D: not determined.

functionalization (Scheme 3). Accordingly, $\mathbf{5 b}$ was treated with ethyl acrylate under ruthenium catalysis to obtain the orthoalkenylated product 8 in $48 \%$ yield. ${ }^{[17]}$ To the best of our knowledge, this is the first example of pyrazoline-directed $\mathrm{C}-\mathrm{H}$ functionalization; this method could find use in library synthesis for lead-optimization in medical chemistry/agrochemical programs. Furthermore, treatment of $\mathbf{5 b}$ with zinc in acetic acid 


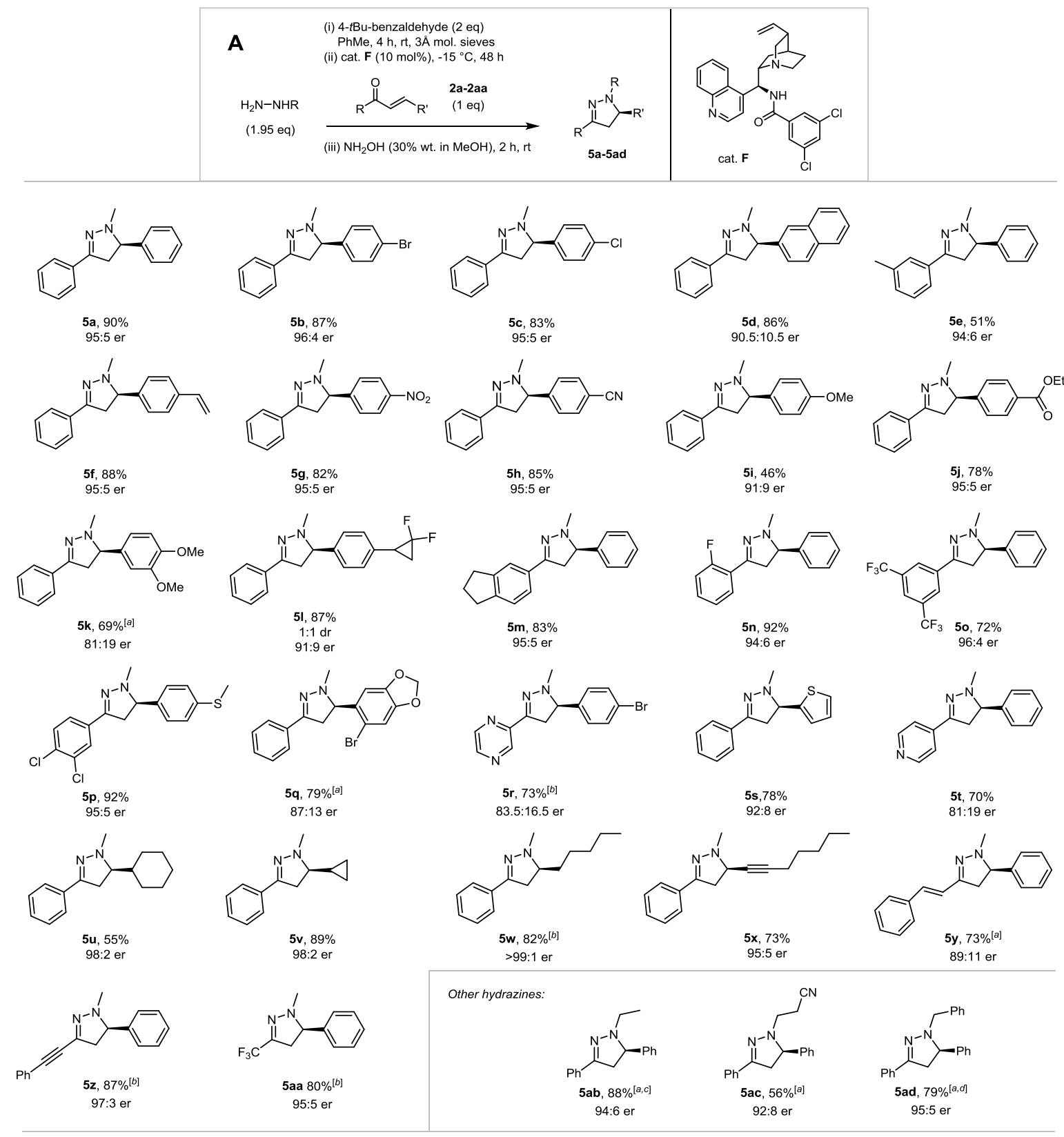

B Large scale synthesis of $\mathbf{5 b}$

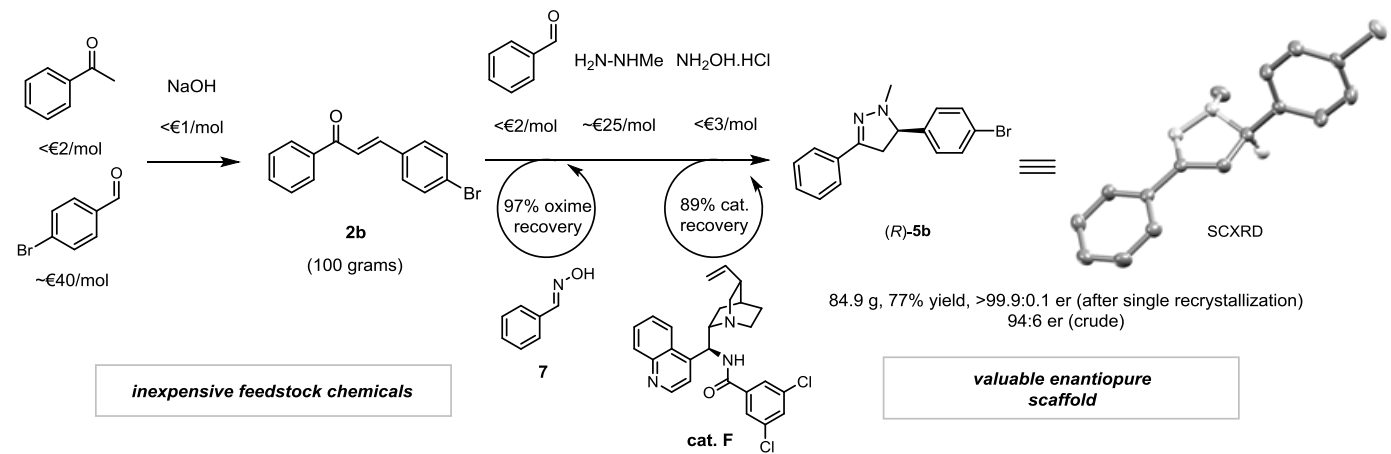

Scheme 2. (A) Scope of aza-Michael addition of in-situ generated 1d to enones 2a-2aa; (B) Large scale synthesis of $\mathbf{5 b}$ (see ESI for details). ${ }^{a}$ performed in $\mathrm{CH}_{2} \mathrm{Cl}_{2} .{ }^{b}$ performed at $-40{ }^{\circ} \mathrm{C}$. ${ }^{\mathrm{C}}$ with EtNHNH${ }_{2} .0 x a$ alate and 1.95 eq DIPEA. ${ }^{d}$ with $\mathrm{BnNHNH} 2.2 \mathrm{HCl}$ and 3.8 eq DIPEA. Stereochemical configuration was assigned by analogy with $(R)-5 \mathbf{b}$ (determined by single crystal $\mathbf{X}$-ray diffraction studies). 


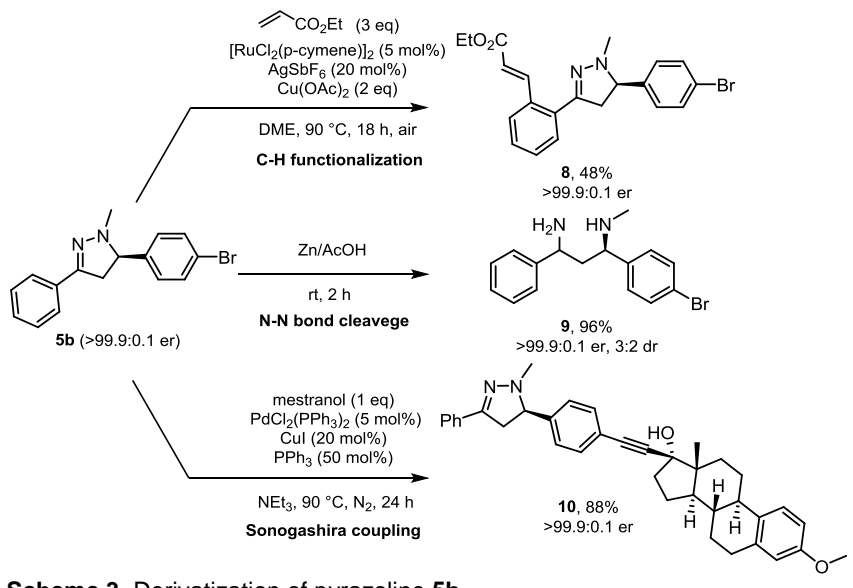

Scheme 3. Derivatization of pyrazoline $5 b$

furnished a 3:2 diastereomeric mixture of the enantioenriched diamine 9 in $96 \%$ yield. Methods to access diamine structures are limited and this example represents the first protocol to obtain optically active 1,3-diamines from pyrazolines. Lastly, Sonogashira coupling with the hormone drug mestranol readily furnished enantiopure alkyne 10 in $88 \%$ yield.

In summary, an efficient platform for the synthesis of enantioenriched 2-pyrazoline scaffolds from beta-substituted enones and monoalkyl-substituted hydrazine-derived hydrazones has been designed and developed. Deployment of a novel cinchonidine-derived bifunctional catalyst in conjunction with an optimized hydrazone derivative allowed good control of reactivity and enantioselectivity in the initial Michael addition step. A staged addition of hydroxylamine then facilitated 2pyrazoline formation after cleavage of the chiral hydrazone intermediate. The new synthetic protocol was found to be compatible with a range of functionalities including esters, nitriles, heterocycles, alkenes and alkynes, and was amenable to decagram scale synthesis. The scalability of this method, coupled with a range of novel late-stage derivatizations has highlighted the synthetic versatility of the reaction products, with potential applications in biomedical and agrochemical contexts.

\section{Acknowledgements}

DJD and CJT wish to thank Bayer AG for generous financial support and Dr. Uwe Döller for helpful discussions. We are grateful to Dr. Jamie Leitch for help in the preparation of this manuscript. Heyao Shi is also thanked for X-ray structure determination of compound $\mathbf{5 b}$, and $\mathrm{Dr}$ Amber $\mathrm{L}$. Thompson and $\mathrm{Dr}$ Kirsten E. Christensen (Oxford Chemical Crystallography) for X-ray mentoring.

Keywords: hydrazone, enantioselective catalysis, pyrazolines, aza-Michael addition, bifunctional catalysis

[1] (a) L. C. Behr, R. Fusco, C. H. Jarboe, Pyrazoles, Pyrazolines, Pyrazolidines, Indazoles and Condensed Rings, The Chemistry of Heterocyclic Compounds 22, Interscience Publishers, New York, 1967; b) L. M. Blair, J. Sperry, J. Nat. Prod. 2013, 76, 794; c) V. Kumar, K. Kaur, G. K. Gupta, A. K. Sharma, Eur. J. Chem. 2013, 69, 735; d) G. Le Goff, J. Ouazzani, J. Bioorg. Med. Chem. 2014, 22, 6529; e) Y.-L. Du, H.-Y. He, M. A. Higgins, K. S. Ryan, Nat. Chem. Bio. 2017, 13, 836.

[2] (a) J. H. Lange, H. K. Coolen, H. H. van Stuiven-berg, J. A. Dijksman, A. H. Herremans, E. Ronken, H. G. Keizer, K. Tipker, A. C. McCreary, W. Veerman, H. C. Wals, B. Stork, P. C. Verveer, A. P. den Hartog, N M. de Jong, T. J. Adolfs, J. Hoogendoorn, C. G. Kruse, J. Med. Chem 2004, 47, 627; b) J. R. Goodell, F. Puig-Basagoiti, B. M. Forshey, P.-Y. Shi, D. M. Ferguson, J. Med. Chem. 2006, 49, 2127; c) P. K. Mykhailiuk, Angew. Chem. Int. Ed. 2015, 54, 6558 .
[3] (a) X. C. Gao, H. Cao, L. Q. Zhang, B.W. Zhang, Y. Cao, C. H. Huang J. Mater. Chem. 1999, 9, 1077; b) A. Gurspy, S. Demirayak, G. Capan, K. Erol, K. Vural, Eur. J. Med. Chem. 2000, 35, 359; c) H. B. Fu, J. N.Yao, J. Am. Chem. Soc. 2001, 123, 1434; d) M. E. Camacho, J. Leon, A. Entrena, G. Velasco, M. D. Carrion, G. Escames, A. Vivo, D. AcunaCastroviejo, M. A. Gallo, A, Espinosa, J. Med. Chem. 2004, 47, 5641; e) X. Zhang, X. Li, G. F. Allan, T. Sbriscia, O. Linton, S. G. Lundeen, Z. Sui, J. Med. Chem. 2007, 50, 3857; f) A. S. Girgis, A. H. Basta, H. ElSaied, M. A. Mohamed, A. H. Bedair, A. S. Salim, R. Soc. Open Sci. 2018, 5, 171964

[4] (a) D. E. Applequist, R. D. Gdanski, J. Org. Chem. 1981, 46, 2502; b) R. S. Padmavathi, R. Venugopal, B. R. Padmaja, Heteroat. Chem. 2003, 14, 155; c) D.-H. Lee, B. S. Jung, S. Jung, J. Song, W. K. Sueng, Tettrahedron Lett., 2005, 46, 7721; d) B. Dipanwita, K.Utpal, K Rajiv, M. Gourhari, Tetrahedron Lett. 2014, 55, 5333; e) D. Havrylyuk O. Roman, R. Lesyk, Eur. J. Med. Chem. 2016, 113, 145

[5] For key publications see: (a) M. P. Sibi, L. M. Stanley, C. P. Jasperse, J. Am. Chem. Soc. 2005, 127, 8276; b) T. Kano, T. Hashimoto, K. Maruoka, J. Am. Chem. Soc. 2006, 128, 2174; c) M. P. Sibi, L. M. Stanley, T. Soeta, Adv. Synth. Catal. 2006, 348, 2371; d) M. P. Sibi, L. M. Stanley, T. Soeta, Adv. Synth. Catal. 2006, 348, 2371; e) M. P. Sibi, L. M. Stanley, T. Soeta, Org. Lett. 2007, 9, 1553; f) L. Gao, G.-S. Hwang, M. Y. Lee, D. H. Ryu, Chem. Commun. 2009, 5460; g) E. Frank, Z. Mucsi, I. Zupko, B. Rethy, G. Falkay, G. Schneider, J. Wolfling, J. Am. Chem. Soc. 2009, 131, 3894; h) J.-R. Chen, W.-R. Dong, M. Candy, F. F. Pan, M. Jörres, C. Bolm, J. Am Chem Soc. 2012, 134, 6924; i) O. Mahé, I. Dez, V. Levachera, J.-F. Brière, Org. Biomol. Chem. 2012, 10 3946; j) X. Kou, Q. Shao, C. Ye, G. Yang, W. Zhang, J. Am. Chem. Soc 2018, 140, 7587 .

[6] S. Kanemasa, T. Kanai, J. Am. Chem. Soc. 2000, 122, 10710.

[7] (a) S. Müller, B. List, Angew. Chem. Int. Ed. 2009, 48, 9975; b) S. Müller, B. List, Synthesis 2010, 13, 2171; c) M. Rueping, M. S. Maji, H. B. Kucuk, I. Atodiresei, Angew. Chem. Int. Ed. 2012, 51, 12864; d) L. Deiana, G.-L. Zhao, H. Leijonmarck, J. Sun, C. W. Lehmann, A. Cordova, ChemistryOpen, 2012, 1, 134; e) M. Fernandez, E. Reyes, J.L. Vicario, D. Badia, L. Carrillo, Adv. Synth. Catal. 2012, 354, 371

[8] O. Mahe, I. Dez, V. Levacher, J.-F. Brière, Angew. Chem. Int. Ed. 2010 $49,7072$.

[9] N. R. Campbell, B. Sun, R. P. Singh, L. Deng, Adv. Synth. Catal. 2011 353,3123

[10] In our initial studies, a wide screen of organocatalysts failed to produce enantioselectivities $>65: 35$ er in the aza-Michael addition of methylhydrazine to $2 a$ (due to a significant background reaction). For more on the use of hydrazines in racemic pyrazoline synthesis see: (a) G.Wu, R. Liang, Y. Chen, L. Wu, Syn. Comm. 2010, 40, 129; b) Z.-P. Lin, J.-T. Li, E-Journal of Chemistry, 2012, 9, 267. For more on the nucleophillicity of hydrazines see: (a) J. M. Garver, S. Gronert, V. M. Bierbaum, J. Am. Chem. Soc. 2011, 133, 13894; b) T. A. Nigst, A Antipova, H. Mayr, J. Org. Chem. 2012, 77, 8142.

[11] For publications on the diverse reactivity of hydrazones in organic synthesis see: (a) M. Sugiura, S. Kobayashi, Angew. Chem. Int. Ed. 2005, 44, 5176; D. Perdicchia, K. A. Jørgensen, J. Org. Chem. 2007 72, 3565; b) E. Li, P. Xie, L. Yang, L. Liang, Y. Huang, Chem. Asian J. 2013, 8, 603; c) M. d. G. Retamosa, E. Matador, D. Monge, J. M. Lassaletta, R. Fernandez, Chem. Eur. J. 2016, 22, 13430; d) Y. Wang Q. Wang, J. Zhu, Angew. Chem. Int. Ed. 2017, 56, 5612.

[12] For publications on bifunctional organocatalysis: (a) T. Okino, Y. Hoashi, Y. Takemoto, J. Am. Chem. Soc. 2003, 125, 12672; b) H. Li, Y. Wang L. Tang, L. Deng, J. Am. Chem. Soc. 2004, 126, 9906; c) T. Okino, Y Hoashi, T. Furukawa, X. Xu, Y. Takemoto, J. Am. Chem. Soc., 2005 127, 119; d) B. Vakulya, S.Varga, A. Csámpai, T. Soós, Org. Lett. 2005 7, 1967; e) J. Ye, D. J Dixon, P. Hynes, Chem. Commun. 2005, 0 4481 ; f) J. Song, Y. Wang, L. Deng, J. Am. Chem. Soc. 2006, 128, 6048; g) S. H. McCooey, S. J. Connon, Angew. Chem. Int. Ed. 2005 44, 6367; h) B.-J. Li, L. Jiang, M. Liu, Y.-C. Chen, L.-S. Ding, Y. Wu Synlett, 2005, 4, 603; i) M. G. Nunez, A. F. M. Farley, D. J. Dixon, J. Am. Chem. Soc. 2013, 135, 16348. For reviews, see: (a) Y. Takemoto, Org. Biomol. Chem. 2005, 3, 4299; b) S. Connon, Chem. Commun 2008, 2499.

[13] See ESI for possible mechanism of formation. For the enantioselective synthesis of such structures, see: W. Wu, X. Yuan, J. Hu, X. Wu, Y. Wei, Z. Liu, J. Lu, J. Ye, Org. Lett. 2013, 15, 4524.

[14] (a) F. Wang, T. Luo, J. Hu, Y. Wang, H. S. Krishnan, P. V. Jog, S. K Ganesh, G. K. S. Prakash, G. A. Olah, Angew. Chem. Int. Ed. 2011, 50, 7153; b) C. J. Thomson, Q. Zhang, N. Al-Maharik, M. Buehl, D. B. Cordes, A. M. Z. Slawin, D. O'Hagan, Chem. Comm. 2018, 54, 8415.

[15] $\mathrm{PhNHNH}_{2}$ afforded only trace amounts of the desired 2-pyrazoline.

[16] 7 could be recycled to benzaldehyde via hydrolysis with $\mathrm{HCl}$ (see ESI).

[17] (a) J. Li, C. Kornhaass, L. Ackermann, Chem. Commun. 2012, 48, 11343; b) J. A. Leitch, P. B. Wilson, C. L. McMullin, M. F. Mahon, Y Bhonoah, I. H. Williams, C. G. Frost, ACS Catal. 2016, 6, 5520. 
Entry for the Table of Contents (Please choose one layout)

Layout 1:

\section{COMMUNICATION}

Text for Table of Contents

((Insert TOC Graphic here))

Layout 2:

\section{COMMUNICATION}

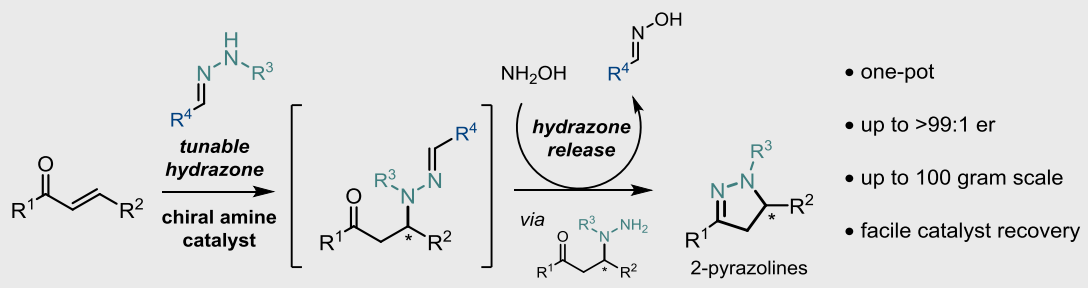

Connor J. Thomson, David M. Barber and Darren J. Dixon*

$1-5$

One-Pot Catalytic Enantioselective Synthesis of 2-Pyrazolines 